\title{
External Validation of the GREAT Score in Turkish Patients with Graves' Hyperthyroidism Treated with the Titration Regimen Method of Antithyroid Drugs: A Multicenter Study
}

Authors

Sayid Shafi Zuhur ${ }^{1}$, Gulsah Elbuken ${ }^{1}$, Ismail Yildiz², Pinar Kadioglu², Selvinaz Erol ${ }^{3}$, Serdar Sahin ${ }^{2}$, Faruk Kilinc ${ }^{4}$, Gulhan Akbaba ${ }^{5}$, Birol Topcu ${ }^{6}$, Yuksel Altuntas ${ }^{3}$

Affiliations

1 Department of Endocrinology and Metabolism, Faculty of Medicine, Namik Kemal University, Tekirdag, Turkey

2 Department of Endocrinology and Metabolism, Cerrahpasa Faculty of Medicine, Istanbul University, Istanbul, Turkey

3 Department of Endocrinology and Metabolism, Health Science University, Sisli Hamidiye Etfal Training and Research Hospital, Istanbul, Turkey

4 Department of Endocrinology and Metabolism, Faculty of Medicine, Firat University, Elazig, Turkey

5 Department of Endocrinology and Metabolism, Faculty of Medicine, Mugla Sitki Kocman University, Mugla, Turkey

6 Department of Biostatistics, Faculty of Medicine, Namik Kemal University, Tekirdag, Turkey

Key words

Graves' disease, relapse, antithyroid drugs, GREAT score

received $\quad 07.05 .2019$

accepted $\quad 01.07 .2019$

Bibliography

DOI https://doi.org/10.1055/a-0974-3991

Published online: 9.9.2019

Horm Metab Res 2019; 51: 627-633

(c) Georg Thieme Verlag KG Stuttgart · New York

ISSN 0018-5043

Correspondence

Dr. Sayid Shafi Zuhur

Department of Endocrinology and Metabolism

Faculty of Medicine

Namik Kemal University

Degirmenaltı Campus

59030 Suleymanpasa

Tekirdag

Turkey

Tel.: + 90/507/1044 480, Fax: + 90/282/2509950

zuhur744@gmail.com

\section{ABSTRACT}

Recently, the Graves' Recurrent Events After Therapy score (GREAT) was proposed as a useful tool to predict relapse before starting antithyroid drugs (ATD) in patients with Graves' disease (GD). Therefore, we intended to assess the validity of the GREAT score in Turkish patients with GD, including patients who experienced a poorly controlled disease (multiple episodes of hyperthyroidism followed by euthyroidism or rarely hypothyroidism) during ATD dose titration. This is a retrospective multicenter study including 517 patients with the first episode of GD who were treated for at least 12 months. The patients were classified as relapse + poorly controlled disease (non-remission) and remission groups. During a median follow-up time of 35 months (12-144 months), 191 (37\%) patients experienced a relapse, 136 (26.3\%) a poorly controlled disease, and 190 (36.7\%) remained in remission. Patients with non-remission disease tended to have significantly higher serum levels of TRAb, fT4, and fT3, and have larger goiter sizes on palpation at baseline, as compared with the remission group. Non-remission disease occurred in 12,35 , and, $53 \%$ of the patients falling into GREAT class I, II, and III, respectively (hazard ratio $2.56,95 \% \mathrm{Cl} 2.02-3.51$, $\mathrm{p}=0.012$, and hazard ratio $3.54,95 \% \mathrm{Cl} 2.12-5.91, \mathrm{p}<0.001$, for GREAT class II and III against class I, respectively). According to our study, the GREAT score is a useful tool to predict the risk of relapse as well as the occurrence of poorly controlled disease before starting treatment with ATDs. 


\section{Introduction}

Graves' disease (GD) is the most common cause of hyperthyroidism in iodine-replete areas of the world [1]. Currently, treatment options for GD include antithyroid drugs (ATD), radioactive iodine (RAI) treatment, and total thyroidectomy [2]. ATDs, including methimazole and propylthiouracil, are currently the first treatment option, particularly in Europe and Asia [2]. However, relapse occurs in $30-60 \%$ of the patients with GD after the withdrawal of ATDs, regardless of the ATD regimen method used [3]. On the other hand, ATDs are also associated with some side effects, which may be severe, and even fatal [4]. Therefore, the selection of patients with a higher probability of long-lasting remission, and the prediction of relapse before the start of ATDs are of paramount importance. Several individual factors, including orbitopathy, smoking, goiter size, severe biochemical hyperthyroidism, and persistent high TRAb levels have been suggested to be associated with a high risk of relapse after ATD withdrawal, but the exact outcome has generally been difficult to predict [5]. Recently, Vos et al. proposed the Graves' Recurrent Events After Therapy score (GREAT and GREAT + ) as a tool to predict relapse before starting ATD in Dutch patients with GD [6]. They concluded that the GREAT score has a great potential for the selection of optimal treatment strategy in individual patients with treatment-naive GD [6]. Subsequently, in a retrospective study, Struja et al. assessed the validity of the GREAT score in Swiss patients with GD [3]. They also concluded that, although not perfect, the GREAT score was a practical and reliable tool to predict the risk of relapse, before the start of ATD treatment in patients with GD [3]. However, before its implication in clinical practice, it should be validated in different patient populations.

The clinical presentation, disease course, and response to ATDs differ among patients with GD. For instance, while a subset of patients with GD enters a full remission after ATD withdrawal, a considerable number of patients may experience several episodes of hyperthyroidism after titration of ATD dose [7]. Likewise, in a minority of patients treated with ATDs, a rare condition consisting of a growing goiter in association with undetectable TSH, relatively low fT4, and high fT3 levels may develop [8]. Moreover, a minority of patients may also experience hypothyroidism from hyperthyroidism or vice versa, even after a minimal change in ATD dose, a condition known as brittle hyperthyroidism $[9,10]$. In general, all of these conditions are known as the poorly controlled disease, and a change in ATD dose is often required for several times during the follow-up period. However, the efficacy of the GREAT score for the prediction of the poorly controlled disease has not been assessed so far. Therefore, in this retrospective multicenter cohort study, we intended to assess the validity of the GREAT score in Turkish patients with $G D$, including a subgroup of patients who experienced a poorly controlled disease (multiple episodes of hyperthyroidism followed by euthyroidism, or rarely hypothyroidism, ensuing after ATD dose titration) during treatment with ATDs.

\section{Patients and Methods}

\section{Patients}

This study includes 517 (374 female and 143 male) patients with the first episode of Graves' disease who were treated with the ti- tration regimen method of ATDs, during the period January 2006January 2018, in 5 endocrinology referral centers across Turkey. Patients $<18$ years old, pregnant patients, ATD treatment $<12$ months, follow-up $<12$ months, patients with subclinical hyperthyroidism, patients who developed any kind of hyperthyroidism during treatment with amiodarone, patients with major side effects of antithyroid drugs, and patients with a history of thyroidectomy or RAl treatment were not included in the study. All patients were classified as relapse + poorly controlled (non-remission) and remission groups, according to the follow-up data. All patients received methimazole at an initial dose of $10-30 \mathrm{mg} /$ day, and methimazole was adjusted to maintenance doses $(2.5-5 \mathrm{mg} /$ day), determined by circulating serum fT 4 and fT3 concentrations. However, patients with minor side effects of methimazole were switched to appropriate doses of propylthiouracil. Generally, the clinical and biochemical evaluation was carried out before the start of treatment, monthly during the first 3 months, subsequently at 3-month intervals, and immediately before treatment withdrawal. Post-treatment follow-up was performed at 3-6-month intervals. GD was defined as clinical hyperthyroidism with or without orbitopathy associated with suppressed or undetectable serum TSH, elevated serum fT4 and/or fT3 levels, diffuse hypoechoic ultrasonographic appearance of the thyroid gland, high TRAb levels or high uptake of $99 \mathrm{~m}-\mathrm{Tc}$ pertechnetate in association with clinical, biochemical, and follow-up findings in patients with normal TRAb titers. Poorly controlled disease in this study was defined as the multiple episodes of clinical and/or biochemical hyperthyroidism followed by euthyroidism and rarely hypothyroidism, ensuing after titration of ATDs during at least one-year follow-up in patients with compliance to treatment. However, to render the patients euthyroid as quickly as possible, the patients with the poorly controlled disease were generally followed-up at monthly intervals after each ATD dose titration. Relapse was defined as overt clinical and biochemical hyperthyroidism after the withdrawal of ATDs. The classification of patients according to the GREAT score was performed as previously reported by Vos et al. [6]. The study protocol was approved by the local ethics committee.

\section{Clinical and laboratory data}

Clinical and laboratory data were obtained through computer records of each center. The following data of each patient at first admission were recorded: The age, gender, goiter size according to the WHO classification (0-III), serum TSH, fT3, fT4, TRAb, anti-TPO, and anti-TG antibody levels, presence or absence of orbitopathy, smoking status, the type of ATD used, date of start and withdrawal of ATD, treatment duration, date of relapse, and application of definitive treatment, if any. Orbitopathy was classified according to Werner's criteria as absent (class 0-1) or present (class 2-6: the presence of inflammatory signs, proptosis, extraocular muscle, and corneal involvement) [11].

Laboratory analysis to detect serum TSH, fT3, fT4, anti-TPO, and anti-TG levels was performed on the day of admission in each study center, after phlebotomy and appropriate centrifugation. The serum of patients to assess TRAb levels were stored at $-20^{\circ} \mathrm{C}$, until were assessed weekly. Serum TSH, fT3, fT4, anti-TPO, and anti-TG levels were assessed by direct chemiluminescence immunoassay method in all centers (Cobas Elecsys, Roche Diagnostics, Manheim, 
Germany, and Siemens, ADVIA Centaur XP Immunoassay System, Tarrytown, NY, USA). Serum TRAb levels were assessed by chemiluminescence immunoassay, TRAb-Fast ELISA, and radioimmunoassay methods (Cobas Elecsys, Roche Diagnostics, Manheim, Germany, Euroimmun AG Seekamp, Lübeck, Germany, and anti-rTSH, RIA kits, Immunotech, Beckman Coulter, Prague, Czech Republic) in different study centers. The normal laboratory reference values for TRAb, as were established by the manufacturers, were $<1.5 \mathrm{IU} / \mathrm{I}$ for the first 2 assay methods, and $<1.75 \mathrm{IU} / \mathrm{I}$ for the last assay method.

\section{Statistical analysis}

In this study, we carried out the same statistical analysis as were previously done in the original and external validation studies of the GREAT score $[3,6]$. As shown in $>$ Table $\mathbf{1}$, we also used the same predetermined cut-off values for the dichotomization of data $[3,6]$. To estimate the association of predetermined risk factors with the non-remission disease, we used Cox proportional hazards regression models. Kaplan-Meier method was used to demonstrate the cumulative rate of remission over follow-up time. The discriminative ability of the GREAT score for prediction of relapse was estimated using the area under the receiver operator curve (AUC). Categorical and continuous variables were analyzed by Pearson's chi-squared test and independent samples t-test (or Mann-Whitney U-test), as appropriate. Analysis of variance (ANOVA) followed by post-hoc analysis (Tamhane T2) was used for multiple comparisons. All significance tests were two-sided, and $p<0.05$ was considered statistically significant. The statistical analysis was performed using PASW Statistics 18 for Windows statistical software package (IBM Corp., Armonk, NY, USA).

\section{Results}

In this study, during a median follow-up time of 35 months (12-144 months) 191 (37\%) patients experienced a relapse, $136(26.3 \%)$ had

- Table 1 Parameters used in the GREAT score and the cut-off values of each parameter.

\begin{tabular}{|c|c|}
\hline Parameter & GREAT score \\
\hline Age & \\
\hline$<40$ & +1 \\
\hline$\geq 40$ & 0 \\
\hline TRAb (U/I) & 0 \\
\hline$<6$ & +1 \\
\hline $6-19.9$ & +2 \\
\hline$>19.9$ & 0 \\
\hline fT4 (pmol/l) & +1 \\
\hline$<40$ & \\
\hline$\geq 40$ & 0 \\
\hline Goiter Size & +2 \\
\hline $0-$ I & \\
\hline II-III & \\
\hline
\end{tabular}

a poorly controlled disease, and 190 (36.7\%) remained in remission. Relapse occurred in $71 \%$ of the patients within the first year after ATD withdrawal and in $29 \%$ more than one year after ATD withdrawal. On the other hand, 86 (63\%), 30 (22\%), and 20 (15\%) patients with the poorly controlled disease were followed-up for 12-24, 25-36, and > 36 months, respectively. However, a permanent euthyroid state could not be achieved in the majority of these patients. Due to the quite similar pretreatment clinical and laboratory characteristics, patients with relapse and poorly controlled disease were combined in a single group. The characteristics of patients classified as remission, relapse and poorly controlled disease as separate groups and in combination as a non-remission group are presented in $>$ Table 2 . In this study, patients in the non-remission group tended to have significantly higher serum levels of TRAb, $\mathrm{fT} 4$, and fT3, and have larger goiter sizes on thyroid palpation, as compared with the remission group ( $\triangleright$ Table 2 ). The non-remission disease was also significantly more frequent in male patients, and patients with orbitopathy, as compared with female patients and patients without orbitopathy $(p<0.001$ for both comparisons, Pearson's chi-squared test). Among 143 male patients, remission and non-remission disease were observed in 31 (21.5\%) and 112 (78.5\%) patients, respectively. Whereas among 374 female patients, remission and non-remission disease were observed in 159 (42.5\%) and 215 (57.5\%) patients, respectively. Likewise, orbitopathy was observed in $36(19 \%)$ and 124 (38\%) patients with remission and non-remission disease, respectively.

Among study patients, 470 (83.2\%) were treated with methimazole and 87 (16.8\%) with propylthiouracil. No major side effect of ATDs was observed among study participants during the follow-up period.

In this study, a gradual increase in the risk of non-remission disease was observed with an increase in the GREAT score. Non-remission disease occurred in 12,35 , and, $53 \%$ of patients falling into GREAT class I, II, and III, respectively (HR 2.56, $95 \% \mathrm{Cl} 2.02-3.51$, $\mathrm{p}=0.012$, and $\mathrm{HR} 3.54,95 \% \mathrm{Cl} 2.12-5.91 \mathrm{p}<0.001)$.

In univariate analysis, significant relationships were found for TRAb, age $<40$ years, FT4 $>40 \mathrm{pmol} / \mathrm{l}$, and goiter sizes II and III with the non-remission disease, whereas no significant relationship was found for fT3 (data not shown). However, in multivariate analysis, a significant association was only found for larger goiter size with the non-remission disease ( $\triangleright$ Table 3 ). The GREAT score showed a fairly good discriminative ability with an AUC of $0.806(95 \% \mathrm{Cl}$ $0.766-0.845)$, for the prediction of non-remission disease. Relapse developed in 4 patients after 72 months of follow-up, of whom 3 were classified as GREAT class I, and 1 as class III. The cumulative rate of remission over 2 years follow-up period is shown in the Kaplan-Meier curve ( $>$ Fig. 1).

\section{Discussion}

Although there is not any best treatment modality for GD yet, the answer to the basic question "which one of the currently available treatments is the best initial treatment option for GD?" has always been controversial. The main reason for this controversy is the high likelihood of relapse occurring after treatment with ATDs, and almost inevitable occurrence of permanent hypothyroidism after total thyroidectomy and RAI treatment. Therefore, to find an ap- 
- Table 2 Characteristics of patients with treatment-naive GD before starting treatment with ATDs.

\begin{tabular}{|c|c|c|c|c|c|c|c|c|}
\hline \multirow{2}{*}{ Parameters } & Relapse & Poorly Controlled & Non-remission ${ }^{\ddagger}$ & Remission & $\mathbf{p}^{1}$ & $\mathbf{p}^{2}$ & $p^{3}$ & $\mathbf{p}^{4}$ \\
\hline & $n=191(37 \%)$ & $n=136(26.3 \%)$ & $n=327(63 \%)$ & $n=190(36.7 \%)$ & & & & \\
\hline Female/Male (\%) & $134 / 57(70.2 / 29.8)$ & $81 / 55(59.6 / 40.4)$ & $215 / 112(65.7 / 34.3)$ & $159 / 31(83.7 / 16.3)$ & _ & _ & _ & - \\
\hline \multicolumn{9}{|c|}{ Parameters of the GREAT score } \\
\hline Age at Diagnosis & $40.8 \pm 12.3$ & $41.7 \pm 13.3$ & $41.2 \pm 12.7$ & $43.3 \pm 12.9$ & 0.78 & 0.14 & 0.53 & 0.07 \\
\hline \multicolumn{9}{|l|}{ Goiter Size } \\
\hline Grade $0-1$ (\%) & $67(35.1)$ & $12(8.8)$ & $79(24)$ & $163(85.8)$ & - & - & - & - \\
\hline Grade II (\%) & $99(51.8)$ & $84(61.8)$ & $183(56)$ & $25(13.2)$ & - & - & - & - \\
\hline Grade III (\%) ${ }^{* *}$ & $25(13.1)$ & $40(29.4)$ & $65(20)$ & $2(1.1)$ & _- & _- & _- & $<0.001$ \\
\hline TRAb (IU/I) & $14.15 \pm 14.20$ & $17.91 \pm 16.91$ & $15.7 \pm 15.5$ & $9.03 \pm 12.24$ & 0.051 & 0.002 & $<0.001$ & $<0.001$ \\
\hline FT4 (pmol/l) & $47.23 \pm 25.74$ & $50.17 \pm 24.03$ & $48.5 \pm 25$ & $40.49 \pm 19.94$ & 0.49 & 0.014 & 0.001 & $<0.001$ \\
\hline \multicolumn{9}{|l|}{ Great Score } \\
\hline Class I ( $0-1$ points) & $32(16.75)$ & $7(5.15)$ & $39(12)$ & $113(59.47)$ & - & - & - & - \\
\hline Class II (2-3 points) & $74(38.75)$ & $41(30.15)$ & $115(35)$ & $60(31.58)$ & - & - & - & - \\
\hline Class III (4-6 points) & $85(44.50)$ & $88(64.70)$ & $173(53)$ & $17(8.91)$ & - & - & - & - \\
\hline \multicolumn{9}{|l|}{ Other parameters } \\
\hline FT3 (pg/ml) & $12.73 \pm 9.68$ & $12.72 \pm 7.67$ & $12.72 \pm 8.9$ & $10.33 \pm 6.18$ & 1.0 & 0.01 & 0.022 & $<0.001$ \\
\hline TSH (IU/I) & $0.009 \pm 0.011$ & $0.007 \pm 0.008$ & $0.008 \pm 0.001$ & $0.010 \pm 0.017$ & 0.50 & 0.59 & 0.11 & 0.02 \\
\hline Anti-TPO (U/I) & $284.6 \pm 327.2$ & $334 \pm 883$ & $305.6 \pm 624.8$ & $205.4 \pm 247$ & 0.67 & 0.31 & 0.076 & 0.05 \\
\hline Anti-TG (U/I) & $329.1 \pm 648.1$ & $341 \pm 796$ & $334.2 \pm 713.5$ & $329 \pm 632.4$ & 0.98 & 1.0 & 0.98 & 0.9 \\
\hline Smoking Status (yes/no) & $77 / 114(40 / 60)$ & $60 / 76(44 / 56)$ & $137 / 190(42 / 58)$ & $75 / 115(39.5 / 60.5)$ & - & - & - & 0.58 \\
\hline Orbitopathy (yes/no) & $67 / 124(35 / 65)$ & $57 / 79(42 / 58)$ & $124 / 203(38 / 62)$ & $36 / 154(19 / 81)$ & _- & _- & _- & $<0.001$ \\
\hline Treatment Duration * & $21.8 \pm 9.5$ & $26.4 \pm 13.27$ & $20.1 \pm 18.1$ & $23.7 \pm 11.4$ & $<0.001$ & $<0.001$ & 0.003 & $<0.001$ \\
\hline Follow-up Duration * & $13.6 \pm 15.2$ & $31.1 \pm 16.86$ & $37.4 \pm 21.3$ & $37.12 \pm 16.89$ & $<0.001$ & 0.037 & 0.017 & 0.038 \\
\hline \multicolumn{9}{|l|}{ Final Treatment Method } \\
\hline ATD (\%) & $94(49.2)$ & $25(18.4)$ & $119(36.4)$ & - & - & - & - & - \\
\hline RAI Treatment (\%) & $52(27.3)$ & $39(28.6)$ & $91(27.8)$ & - & - & - & - & - \\
\hline Total Thyroidectomy (\%) & $45(23.5)$ & $72(53)$ & $117(35.8)$ & - & - & - & - & - \\
\hline
\end{tabular}

ATD: Antithyroid drugs; RAl: Radioactive iodine. $\mathrm{p}^{1}$ : The difference between patients with relapse and the poorly controlled disease; $\mathrm{p}^{2}$ : The difference between patients with relapse and remission; $\mathrm{p}^{3}$ : The difference between patients with the poorly controlled disease and remission $\mathrm{P}^{4}$ : The difference between patients with relapse + poorly controlled disease and remission. $\ddagger$ Relapse + poorly controlled disease, " Months.

propriate answer to that question, the prediction of relapse before starting treatment with ATDs has become an active area of research.

Several studies have been conducted so far to assess the risk of relapse in patients with GD before starting treatment with ATDs. Until recently, different individual clinical, biochemical, genetic, and behavioral parameters, including goiter size, the presence of Graves' orbitopathy, severe biochemical hyperthyroidism, TRAb level, HLA genotype, and smoking status have been assessed to predict the risk of relapse $[5,12,13]$. But, no studies have yet found the best individual parameter to predict the risk of relapse before starting ATDs.

Recently, Vos et al. proposed the GREAT and GREAT + scores, a combination of clinical and laboratory parameters, to predict the risk of relapse before the start of ATDs in patients with GD [6]. They found a relapse rate of 16,44 , and $68 \%$ in patients classified as
GREAT class I, II and III, respectively [6]. Afterward, Struja et al. performed an external validation of the GREAT score [3]. In their study, the distribution of relapse according to GREAT class I, II and III was $33.8,59.4$ and $73.6 \%$, respectively (HR 1.79, $95 \% \mathrm{Cl} 1.42-2.27$ and HR 2.24, $95 \% \mathrm{Cl} 1.64,95 \% \mathrm{Cl} 1.64-3.06)$. In our study, the corresponding distribution of non-remission disease according to GREAT class I, II, and III was 12,35 , and $53 \%$, respectively, and was in agreement with that reported in the original study of Vos et al. [6].

One of the main differences of our study from the original and external validation studies is the inclusion of a subgroup of patients with a poorly controlled disease. This situation is troublesome for both the patient and the physician and requires frequent clinical and biochemical control. Unfortunately, this subgroup of patients has not been included in most studies predicting the outcome of ATDs. Although the inclusion of this subgroup of patients in our study may have contributed to a lower rate of remission, we believe 
D Table 3 The risk of non-remission disease according to GREAT score determined by uni- and multivariate analysis.

\begin{tabular}{|c|c|c|c|c|c|}
\hline Parameter & Non-remission $¥(\%)$ & $\operatorname{HR}(95 \% \mathrm{Cl}) *$ & p-Value & $\operatorname{HR}(95 \% \mathrm{Cl})^{* *}$ & p-Value \\
\hline \multicolumn{6}{|l|}{ Age } \\
\hline$\geq 40$ & $58.3(148 / 254)$ & \multicolumn{2}{|c|}{ Ref. } & \multicolumn{2}{|c|}{ Ref } \\
\hline$<40$ & $68.1(179 / 263)$ & $1.51(1.22-1.90)$ & 0.015 & $1.22(0.94-1.58)$ & 0.128 \\
\hline \multicolumn{6}{|l|}{ TRAb (IU/I) } \\
\hline$<6$ & $44.2(91 / 206)$ & \multicolumn{2}{|c|}{ Ref. } & \multicolumn{2}{|c|}{ Ref } \\
\hline $6-19.9$ & $72.8(147 / 202)$ & $1.57(1.14-2.17)$ & 0.006 & $1.26(0.82-1.92)$ & 0.279 \\
\hline$>19.9$ & $81.7(89 / 109)$ & $2.12(1.32-3.42)$ & 0.002 & $1.01(0.76-1.36)$ & 0.897 \\
\hline \multicolumn{6}{|c|}{ fT4 (pmol/l) } \\
\hline$<40$ & $57(150 / 263)$ & \multicolumn{2}{|c|}{ Ref. } & \multicolumn{2}{|c|}{ Ref } \\
\hline$\geq 40$ & $69.7(177 / 254)$ & $1.28(1.04-1.60)$ & 0.023 & $1.10(0.84-1.43)$ & 0.478 \\
\hline \multicolumn{6}{|l|}{ Goiter size } \\
\hline $0-1$ & $32.6(79 / 242)$ & \multicolumn{2}{|c|}{ Ref. } & \multicolumn{2}{|c|}{ Ref. } \\
\hline II-III & $90.1(248 / 275)$ & $4.2(3.24-5.46)$ & $<0.001$ & $2.87(1.76-4.65)$ & $<0.001$ \\
\hline
\end{tabular}

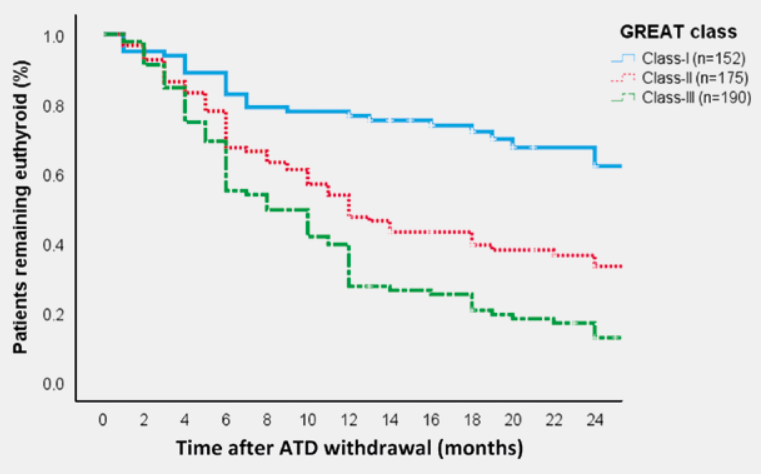

- Fig. 1 Kaplan-Meier curve displaying cumulative remission rate over 2 years of follow-up according to the GREAT score.

that the inclusion of this subgroup of patients reflects the daily practice more accurately. As demonstrated in $>$ Table 2, patients with the poorly controlled disease had a borderline higher mean TRAb levels compared to patients with relapse, otherwise, they shared the same clinical and biochemical features with patients who experienced a relapse. Hence, relapse and the poorly controlled disease may be the two sides of the same coin. While the majority of patients with the same features develop a relapse after ATD withdrawal, another minority may develop a poorly controlled disease during treatment with ATDs. That is why patients with relapse and those with the poorly controlled disease were combined in a single group in our study. However, in a study carried out by Kim et al. in Korean patients with GD, the patients with homogeneously distributed TRAb epitope on TSH receptor have failed to discontinue ATD during 24 months of treatment due to the several episodes of hyperthyroidism ensued after ATD dose titration, compared to patients with heterogeneously distributed TRAb epitopes on TSH receptor [14]. Their study suggests that some molecular pathways may also contribute to a poor response to ATDs. Although there is not enough data to explain the characteristics and frequency of poorly controlled cases, the number of patients with the poorly controlled disease among the participants of our study seems to be high. This situation might be multifactorial, including the larger goiter sizes of patients with Graves' disease, the geographical difference in iodine consumption, the strict inclusion criteria as well as the ethnic and genetic background of the study population. For instance, while CTLA4-49 polymorphism was not associated with relapse in the original study of Vos et al. [6], it was suggested as a risk factor for relapse in Turkish patients with GD [15]. However, there is no study so far to explain the exact cause of the high number of poorly controlled cases during ATD treatment in our country.

The results of uni- and multivariate analysis in our study were also in agreement with that reported by Vos et al. [6]. In univariate analysis, significant associations were found for TRAb levels $>6 \mathrm{IU}$, younger age at disease onset, higher fT 4 levels, and larger goiter size with the non-remission disease, while in multivariate analysis, a significant association was only found for larger goiter size ( $\vee$ Table 3). Therefore, according to the results of the present study, the GREAT score showed quite good external validity in Turkish patients with GD. The GREAT score could not only predict the risk of relapse but could also predict the occurrence of poorly controlled disease during treatment with ATD.

Although an association between smoking status with relapse and poorly controlled disease was not found in our study, the non-remission disease was significantly more frequent in male patients and patients with orbitopathy, as compared to female patients and patients without orbitopathy. As illustrated in the results section as well as in $>$ Table 2 , roughly $2 / 3$ of patients were female 
and $1 / 3$ had orbitopathy. The relationship between gender and relapse of GD is controversial. In studies conducted by Vitti et al. and Allahabadia et al. the relapse of GD was significantly more common in male compared to female patients [16, 17]. However, in a recent systematic review and meta-analysis, male sex was not associated with relapse of GD [5]. The results of the studies investigating the association between Graves' orbitopathy and relapse is also controversial. Some studies have found a relationship between severe orbitopathy and relapse [18]. In the aforementioned systematic review and meta-analysis, an association was also found between orbitopathy and relapse, while in the studies conducted by Vos et al. and Struja et al., no relationship was found between orbitopathy and relapse of GD $[3,6]$. Therefore, the association between male sex and orbitopathy with relapse should be investigated in further studies. However, as reported previously, we also could not find a relationship between smoking status, serum fT3, anti-TPO, and anti-TG antibodies with relapse [3,6].

Our study has several limitations. First of all, given its retrospective study design, it is subject to reporting bias. Second, a genetic analysis could not be performed in patients classified as GREAT class II. Third, in our study, we included patients with $>12$ months follow-up, while Struja et al. included patients with $>24$ months follow-up in their study [6]. However, only $10 \%$ of the patients included in our study were followed-up for $<18$ months, and the majority of patients were followed up for $>24$ months. Keeping in mind the high frequency of relapse during the first year after ATD withdrawal, and the low number of patients with $<18$ months follow-up, we don't believe that our results may have been affected by this negligible difference. And finally, the treatment duration and regimen in our study is different from the original study of Vos et al. [6]. While Vos et al. treated the patients for 1 year with the block replace regimen, the patients with relapse and poorly controlled disease in our study were treated for $21.8 \pm 9.5$ and $26.4 \pm 13.27$ months with the titration regimen, respectively. This discrepancy is mostly due to the retrospective design of our study. In Turkey, we generally treat the patients with GD for an extended period of time, before irreversible definitive treatment ensued. Nevertheless, as a Cochrane database study demonstrated that the treatment duration > 18 months has no additional benefit on the remission rate [19], we do not believe that our study results might have been influenced by different treatment duration. The similarity between the predictive performance of the GREAT score in our study and the original study support our assertion as well. Although the block-replace regimen is associated with a slightly higher rate of remission (OR 0.86, $95 \% \mathrm{Cl} 0.68-1.08$ ), it is also associated with a higher frequency of adverse events [19]. Therefore, we generally prefer the titration regimen to avoid adverse events. Considering the small effect of treatment regimen on treatment outcome, we also do not believe that our study results might have been affected by different treatment regimen. On the other hand, the strength of this study derives from the inclusion of a relatively high number of patients with appropriate laboratory, treatment, and in a majority, long term follow-up. Furthermore, it is the first study to report that the GREAT score can also predict the occurrence of the poorly controlled disease before starting ATDs. For the reasons mentioned above, our study population is an evident example of daily practice in our country and probably abroad.

\section{Conclusion}

According to the present multicenter cohort study, The GREAT score is a useful tool to predict the risk of relapse before starting ATD in patients with GD. The risk of relapse is gradually increased from GREAT class I through class III. The GREAT score is also a helpful tool to predict the occurrence of the poorly controlled disease during treatment with ATDs. Therefore, the GREAT score could be used in clinical practice to predict the outcome of treatment with ATDs in patients with treatment-naive GD.

\section{Conflict of Interest}

The authors declare that they have no conflict of interest.

References

[1] Ross DS, Burch HB, Cooper D et al. 2016 American Thyroid Association guidelines for diagnosis and management of hyperthyroidism and other causes of thyrotoxicosis. Thyroid 2016; 26: 1343-1421

[2] Kahaly G], Bartalena L, Hegedüs L et al. 2018 European Thyroid Association guideline for the management of Graves' hyperthyroidism. Eur Thyroid J 2018; 7: 167-186

[3] Struja T, Kaeslin M, Boesiger F et al. External validation of the GREAT score to predict relapse risk in Graves' disease: Results from a multicenter, retrospective study with 741 patients. Eur J Endocrinol 2017; 176: 413-419

[4] Cooper DS. Antithyroid drugs. N Engl J Med 2005; 352: 905-917

[5] Struja T, Fehlberg H, Kutz A et al. Can we predict relapse in Graves' disease? Results from a systematic review and meta-analysis. Eur J Endocrinol 2017; 176: 87-97

[6] Vos XG, Endert E, Zwinderman AH et al. Predicting the risk of recurrence before the start of antithyroid drug therapy in patients with Graves' hyperthyroidism. J Clin Endocrinol Metab 2016; 101: 1381-1389

[7] Kruljac I, Solter D, Vrkljan AM et al. Remission of Graves' disease is not related to early restoration of euthyroidism with high-dose methimazole therapy. Endocr Res 2015; 40: 25-28

[8] Wenzel KW, Lente JR. Syndrome of persisting thyroid stimulating immunoglobulins and growth promotion of goiter combined with low thyroxine and high triiodothyronine serum levels in drug-treated Graves' disease. J Endocrinol Invest 1983; 6: 389-394

[9] Marino M, Latrofa FMenconi et al. An update on the medical treatment of Graves' hyperthyroidism. J Endocrinol Invest 2014; 37: 1041-1048

[10] Laurberg P, Krejbjerg A, Anderson SL. Relapse following antithyroid drug therapy for Graves' hyperthyroidism. Curr Opin Endocrinol Diabetes Obes 2014; 21: 415-421

[11] Werner SC. Modification of the classification of the eye changes of Graves' disease: Recommendations of the ad hoc committee of the American Thyroid Association. J Clin Endocrinol Metab 1977; 44: 203-204

[12] Sundaresh V, Brito JP, Wang Z et al. Comparative effectiveness of therapies for Graves' hyperthyroidism: A systematic review and network meta-analysis. J Clin Endocrinol Metab 2013; 98: 3671-3677

[13] Piantanida E, Lai A, Sassi L et al. Outcome prediction of treatment of graves' hyperthyroidism with antithyroid drugs. Horm Metab Res 2003; 47: 767-772 
[14] Kim TY, Park Y], Park D] et al. Epitope heterogeneity of thyroid-stimulating antibodies predicts long-term outcome in Graves' patients treated with antithyroid drugs. J Clin Endocrinol Metab 2003; 88: 117-124

[15] Tanrikulu S, Erbil Y, Ademoglu E et al. The predictive value of CTLA-4 and Tg polymorphisms in the recurrence of Graves' disease after antithyroid withdrawal. Endocrine 2006; 30: 377-381

[16] Vitti P, Rago T, Chiovato L et al. Clinical features of patients with Graves' disease undergoing remission after antithyroid drug treatment. Thyroid 1997; 7: 369-375
[17] Allahabadia A, Daykin J, Holder RL et al. Ag, and gender predict the outcome of treatment for Graves' hyperthyroidism. J Clin Endocrinol Metab 2000; 85: 1038-1042

[18] Eckstein AK, Lax H, Lösch C et al. Patients with severe Graves' ophthalmopathy have a higher risk of relapsing hyperthyroidism and are unlikely to remain in remission. Clin Endocrinol (Oxf) 2007; 67: 607-612

[19] Abraham P, Avenell A, McGeoch SC et al. Antithyroid drug regimen for treating Graves' hyperthyroidism. Cochrane Database Syst Re 2010; 20: CD003420. doi:10.1002/14651858.CD003420.pub4 